\title{
Upper bound of the third Hankel determinant for a class of analytic functions related with lemniscate of Bernoulli
}

\section{Mohsan Raza ${ }^{1 *}$ and Sarfraz Nawaz Malik²}

\section{"Correspondence:}

mohsan976@yahoo.com

'Department of Mathematics,

G.C. University Faisalabad,

Faisalabad, Pakistan

Full list of author information is

available at the end of the article

\begin{abstract}
In this paper, the upper bound of the Hankel determinant $H_{3}(1)$ for a subclass of analytic functions associated with right half of the lemniscate of Bernoulli $\left(x^{2}+y^{2}\right)^{2}-2\left(x^{2}-y^{2}\right)=0$ is investigated.

MSC: $30 C 45 ; 30 C 50$
\end{abstract}

Keywords: starlike functions; subordination; lemniscate of Bernoulli; Toeplitz determinants; Hankel determinants

\section{Introduction and preliminaries}

Let $A$ be the class of functions $f$ of the form

$$
f(z)=z+\sum_{n=2}^{\infty} a_{n} z^{n}
$$

which are analytic in the open unit $\operatorname{disk} E=\{z:|z|<1\}$. A function $f$ is said to be subordinate to a function $g$, written as $f \prec g$, if there exists a Schwartz function $w$ with $w(0)=0$ and $|w(z)|<1$ such that $f(z)=g(w(z))$. In particular, if $g$ is univalent in $E$, then $f(0)=g(0)$ and $f(E) \subset g(E)$.

Let $P$ denote the class of analytic functions $p$ normalized by

$$
p(z)=1+\sum_{n=1}^{\infty} p_{n} z^{n}
$$

such that $\operatorname{Re} p(z)>0$. Let $S L^{*}$ be the class of functions defined by

$$
S L^{*}=\left\{f \in A:\left|\left(\frac{z f^{\prime}(z)}{f(z)}\right)^{2}-1\right|<1\right\}, \quad z \in E .
$$

Thus a function $f \in S L^{*}$ is such that $\frac{z f^{\prime}(z)}{f(z)}$ lies in the region bounded by the right half of the lemniscate of Bernoulli given by the relation $\left|w^{2}-1\right|<1$. It can easily be seen that $f \in S L^{*}$ if it satisfies the condition

$$
\frac{z f^{\prime}(z)}{f(z)} \prec \sqrt{1+z}, \quad z \in E .
$$


This class of functions was introduced by Sokół and Stankiewicz [1] and further investigated by some authors. For details, see [2, 3].

Noonan and Thomas [4] have studied the $q$ th Hankel determinant defined as

$$
H_{q}(n)=\left|\begin{array}{cccc}
a_{n} & a_{n+1} & \cdots & a_{n+q-1} \\
a_{n+1} & a_{n+2} & \cdots & a_{n+q-2} \\
\vdots & \vdots & \vdots & \vdots \\
a_{n+q-1} & a_{n+q-2} & \cdots & a_{n+2 q-2}
\end{array}\right|,
$$

where $n \geq 1$ and $q \geq 1$. The Hankel determinant plays an important role in the study of singularities; for instance, see [5, p.329] and Edrei [6]. This is also important in the study of power series with integral coefficients [5, p.323] and Cantor [7]. For the use of the Hankel determinant in the study of meromorphic functions, see [8], and various properties of these determinants can be found in [9, Chapter 4]. It is well known that the Fekete-Szegö functional $\left|a_{3}-a_{2}^{2}\right|=H_{2}(1)$. This functional is further generalized as $\left|a_{3}-\mu a_{2}^{2}\right|$ for some $\mu$ (real as well as complex). Fekete and Szegö gave sharp estimates of $\left|a_{3}-\mu a_{2}^{2}\right|$ for $\mu$ real and $f \in S$, the class of univalent functions. It is a very great combination of the two coefficients which describes the area problems posted earlier by Gronwall in 1914-15. Moreover, we also know that the functional $\left|a_{2} a_{4}-a_{3}^{2}\right|$ is equivalent to $H_{2}(2)$. The qth Hankel determinant for some subclasses of analytic functions was recently studied by Arif et al. [10] and Arif et al. [11]. The functional $\left|a_{2} a_{4}-a_{3}^{2}\right|$ has been studied by many authors, see [1214]. Babalola [15] studied the Hankel determinant $H_{3}(1)$ for some subclasses of analytic functions. In the present investigation, we determine the upper bounds of the Hankel determinant $H_{3}(1)$ for a subclass of analytic functions related with lemniscate of Bernoulli by using Toeplitz determinants.

We need the following lemmas which will be used in our main results.

Lemma 1.1 [16] Let $p \in P$ and of the form (1.2). Then

$$
\left|p_{2}-v p_{1}^{2}\right| \leq \begin{cases}-4 v+2, & v<0 \\ 2, & 0 \leq v \leq 1 \\ 4 v-2, & v>1\end{cases}
$$

When $v<0$ or $v>1$, the equality holds if and only if $p(z)$ is $\frac{1+z}{1-z}$ or one of its rotations. If $0<v<1$, then the equality holds if and only if $p(z)=\frac{1+z^{2}}{1-z^{2}}$ or one of its rotations. If $v=0$, the equality holds if and only if $p(z)=\left(\frac{1}{2}+\frac{\eta}{2}\right) \frac{1+z}{1-z}+\left(\frac{1}{2}-\frac{\eta}{2}\right) \frac{1-z}{1+z}(0 \leq \eta \leq 1)$ or one of its rotations. If $v=1$, the equality holds if and only if $p$ is the reciprocal of one of the functions such that the equality holds in the case of $v=0$. Although the above upper bound is sharp, when $0<v<1$, it can improved as follows:

$$
\left|p_{2}-v p_{1}^{2}\right|+v\left|p_{1}\right|^{2} \leq 2 \quad(0<v \leq 1 / 2)
$$

and

$$
\left|p_{2}-v p_{1}^{2}\right|+(1-v)\left|p_{1}\right|^{2} \leq 2 \quad(1 / 2<v \leq 1) .
$$


Lemma 1.2 [16] If $p(z)=1+p_{1} z+p_{2} z^{2}+\cdots$ is a function with positive real part in $E$, then for $v$ a complex number

$$
\left|p_{2}-v p_{1}^{2}\right| \leq 2 \max (1,|2 v-1|)
$$

This result is sharp for the functions

$$
p(z)=\frac{1+z^{2}}{1-z^{2}}, \quad p(z)=\frac{1+z}{1-z} .
$$

Lemma 1.3 [17] Let $p \in P$ and of the form (1.2). Then

$$
2 p_{2}=p_{1}^{2}+x\left(4-p_{1}^{2}\right)
$$

for some $x,|x| \leq 1$, and

$$
4 p_{3}=p_{1}^{3}+2\left(4-p_{1}^{2}\right) p_{1} x-\left(4-p_{1}^{2}\right) p_{1} x^{2}+2\left(4-p_{1}^{2}\right)\left(1-|x|^{2}\right) z
$$

for some $z,|z| \leq 1$.

\section{Main results}

Although we have discussed the Hankel determinant problem in the paper, the first two problems are specifically related with the Fekete-Szegö functional, which is a special case of the Hankel determinant.

Theorem 2.1 Let $f \in S L^{*}$ and of the form (1.1). Then

$$
\left|a_{3}-\mu a_{2}^{2}\right| \leq \begin{cases}\frac{1}{16}(1-4 \mu), & \mu<-\frac{3}{4} \\ \frac{1}{4}, & -\frac{3}{4} \leq \mu \leq \frac{5}{4} \\ \frac{1}{16}(4 \mu-1), & \mu>\frac{5}{4}\end{cases}
$$

Furthermore, for $-\frac{3}{4}<\mu \leq \frac{1}{4}$,

$$
\begin{aligned}
& \qquad\left|a_{3}-\mu a_{2}^{2}\right|+\frac{1}{4}(4 \mu+3)\left|a_{2}\right|^{2} \leq \frac{1}{4}, \\
& \text { and for } \frac{1}{4}<\mu \leq \frac{5}{4} \\
& \qquad\left|a_{3}-\mu a_{2}^{2}\right|+\frac{1}{4}(5-4 \mu)\left|a_{2}\right|^{2} \leq \frac{1}{4} .
\end{aligned}
$$

These results are sharp.

Proof If $f \in S L^{*}$, then it follows from (1.3) that

$$
\frac{z f^{\prime}(z)}{f(z)} \prec \phi(z),
$$


where $\phi(z)=\sqrt{1+z}$. Define a function

$$
p(z)=\frac{1+w(z)}{1-w(z)}=1+p_{1} z+p_{2} z^{2}+\cdots .
$$

It is clear that $p \in P$. This implies that

$$
w(z)=\frac{p(z)-1}{p(z)+1} .
$$

From (2.1), we have

$$
\frac{z f^{\prime}(z)}{f(z)}=\phi(w(z))
$$

with

$$
\phi(w(z))=\left(\frac{2 p(z)}{p(z)+1}\right)^{\frac{1}{2}} .
$$

Now

$$
\left(\frac{2 p(z)}{p(z)+1}\right)^{\frac{1}{2}}=1+\frac{1}{4} p_{1} z+\left[\frac{1}{4} p_{2}-\frac{5}{32} p_{1}^{2}\right] z^{2}+\left[\frac{1}{4} p_{3}-\frac{5}{16} p_{1} p_{2}+\frac{13}{128} p_{1}^{3}\right] z^{3}+\cdots
$$

Similarly,

$$
\frac{z f^{\prime}(z)}{f(z)}=1+a_{2} z+\left[2 a_{3}-a_{2}^{2}\right] z^{2}+\left[3 a_{4}-3 a_{2} a_{3}+a_{2}^{3}\right] z^{3}+\cdots
$$

Therefore

$$
\begin{aligned}
& a_{2}=\frac{1}{4} p_{1}, \\
& a_{3}=\frac{1}{8} p_{2}-\frac{3}{64} p_{1}^{2}, \\
& a_{4}=\frac{1}{12} p_{3}-\frac{7}{96} p_{1} p_{2}+\frac{13}{768} p_{1}^{2} .
\end{aligned}
$$

This implies that

$$
\left|a_{3}-\mu a_{2}^{2}\right|=\frac{1}{8}\left|p_{2}-\frac{1}{8}(4 \mu+3) p_{1}^{2}\right| .
$$

Now, using Lemma 1.1, we have the required result.

The results are sharp for the functions $K_{i}(z), i=1,2,3,4$, such that

$$
\begin{array}{ll}
\frac{z K_{1}^{\prime}(z)}{K_{1}(z)}=\sqrt{1+z} & \text { if } \mu<-\frac{3}{4} \text { or } \mu>\frac{5}{4}, \\
\frac{z K_{2}^{\prime}(z)}{K_{2}(z)}=\sqrt{1+z^{2}} & \text { if }-\frac{3}{4}<\mu<\frac{5}{4},
\end{array}
$$




$$
\begin{array}{ll}
\frac{z K_{3}^{\prime}(z)}{K_{3}(z)}=\sqrt{1+\Phi(z)} & \text { if } \mu=-\frac{3}{4}, \\
\frac{z K_{4}^{\prime}(z)}{K_{4}(z)}=\sqrt{1-\Phi(z)} & \text { if } \mu=\frac{5}{4},
\end{array}
$$

where $\Phi(z)=\frac{z(z+\eta)}{1+\eta z}$ with $0 \leq \eta \leq 1$.

Theorem 2.2 Let $f \in S L^{*}$ and of the form (1.1). Then for a complex number $\mu$,

$$
\left|a_{3}-\mu a_{2}^{2}\right| \leq \frac{1}{4} \max \left\{1 ;\left|\mu-\frac{1}{4}\right|\right\} .
$$

Proof Since

$$
\left|a_{3}-\mu a_{2}^{2}\right|=\frac{1}{8}\left|p_{2}-\frac{1}{8}(4 \mu+3) p_{1}^{2}\right|,
$$

therefore, using Lemma 1.2, we get the result. This result is sharp for the functions

$$
\frac{z f^{\prime}(z)}{f(z)}=\sqrt{1+z}
$$

or

$$
\frac{z f^{\prime}(z)}{f(z)}=\sqrt{1+z^{2}}
$$

For $\mu=1$, we have $H_{2}(1)$.

Corollary 2.3 Let $f \in S L^{*}$ and of the form (1.1). Then

$$
\left|a_{3}-a_{2}^{2}\right| \leq \frac{1}{4}
$$

Theorem 2.4 Let $f \in S L^{*}$ and of the form (1.1). Then

$$
\left|a_{2} a_{4}-a_{3}^{2}\right| \leq \frac{1}{16} .
$$

Proof From (2.2), (2.3) and (2.4), we obtain

$$
\begin{aligned}
a_{2} a_{4}-a_{3}^{2} & =\frac{1}{48}\left(p_{1} p_{3}-\frac{7}{8} p_{1}^{2} p_{2}+\frac{13}{64} p_{1}^{4}\right)-\left(\frac{1}{8} p_{2}-\frac{3}{64} p_{1}^{2}\right)^{2} \\
& =\frac{1}{48} p_{1} p_{3}-\frac{1}{64} p_{2}^{2}-\frac{5}{768} p_{1}^{2} p_{2}+\frac{25}{12,288} p_{1}^{4} \\
& =\frac{1}{12,288}\left(256 p_{1} p_{3}-192 p_{2}^{2}-80 p_{1}^{2} p_{2}+25 p_{1}^{4}\right) .
\end{aligned}
$$

Putting the values of $p_{2}$ and $p_{3}$ from Lemma 1.3, we assume that $p>0$, and taking $p_{1}=p \in$ $[0,2]$, we get

$$
\begin{aligned}
\left|a_{2} a_{4}-a_{3}^{2}\right|= & \frac{1}{12,288} \mid 64 p_{1}\left\{p_{1}^{3}+2\left(4-p_{1}^{2}\right) p_{1} x-\left(4-p_{1}^{2}\right) p_{1} x^{2}+2\left(4-p_{1}^{2}\right)\left(1-|x|^{2}\right) z\right\} \\
& -48\left\{p_{1}^{2}+x\left(4-p_{1}^{2}\right)\right\}^{2}-40 p_{1}^{2}\left\{p_{1}^{2}+x\left(4-p_{1}^{2}\right)\right\}+25 p_{1}^{4} \mid .
\end{aligned}
$$


After simple calculations, we get

$$
\begin{aligned}
\left|a_{2} a_{4}-a_{3}^{2}\right|= & \frac{1}{12,288} \mid 41 p^{4}-8\left(4-p^{2}\right) p^{2} x-128\left(4-p^{2}\right)\left(1-|x|^{2}\right) z \\
& +x^{2}\left(4-p^{2}\right)\left(64 p^{2}+48\right)\left(4-p^{2}\right) \mid .
\end{aligned}
$$

Now, applying the triangle inequality and replacing $|x|$ by $\rho$, we obtain

$$
\begin{aligned}
\left|a_{2} a_{4}-a_{3}^{2}\right| & \leq \frac{1}{12,288}\left[41 p^{4}+128\left(4-p^{2}\right)+8\left(4-p^{2}\right) p^{2} \rho+\rho^{2}\left(4-p^{2}\right)\left(16 p^{2}+64\right)\right] \\
& =F(p, \rho) \quad \text { (say). }
\end{aligned}
$$

Differentiating with respect to $\rho$, we have

$$
\frac{\partial F(p, \rho)}{\partial \rho}=\frac{1}{12,288}\left[8\left(4-p^{2}\right) p^{2}+2 \rho\left(4-p^{2}\right)\left(16 p^{2}+64\right)\right] .
$$

It is clear that $\frac{\partial F(p, \rho)}{\partial \rho}>0$, which shows that $F(p, \rho)$ is an increasing function on the closed interval $[0,1]$. This implies that maximum occurs at $\rho=1$. Therefore $\max F(p, \rho)=F(p, 1)=$ $G(p)$ (say). Now

$$
G(p)=\frac{1}{12,288}\left[17 p^{4}-96 p^{2}+768\right] .
$$

Therefore

$$
G^{\prime}(p)=\frac{1}{12,288}\left[68 p^{3}-192 p\right]
$$

and

$$
G^{\prime \prime}(p)=\frac{1}{12,288}\left[204 p^{2}-192\right]<0
$$

for $p=0$. This shows that maximum of $G(p)$ occurs at $p=0$. Hence, we obtain

$$
\begin{aligned}
\left|a_{2} a_{4}-a_{3}^{2}\right| & \leq \frac{768}{12,288} \\
& =\frac{1}{16} .
\end{aligned}
$$

This result is sharp for the functions

$$
\frac{z f^{\prime}(z)}{f(z)}=\sqrt{1+z}
$$

or

$$
\frac{z f^{\prime}(z)}{f(z)}=\sqrt{1+z^{2}}
$$

Theorem 2.5 Let $f \in S L^{*}$ and of the form (1.1). Then

$$
\left|a_{2} a_{3}-a_{4}\right| \leq \frac{1}{6}
$$


Proof Since

$$
\begin{aligned}
& a_{2}=\frac{1}{4} p_{1}, \\
& a_{3}=\frac{1}{8} p_{2}-\frac{3}{64} p_{1}^{2}, \\
& a_{4}=\frac{1}{12} p_{3}-\frac{7}{96} p_{1} p_{2}+\frac{13}{768} p_{1}^{2} .
\end{aligned}
$$

Therefore, by using Lemma 1.3, we can obtain

$$
\left|a_{2} a_{3}-a_{4}\right| \leq \frac{1}{768}\left\{7 p^{3}+8 p \rho\left(4-p^{2}\right)+32\left(4-p^{2}\right)+16 \rho^{2}(p-2)\left(4-p^{2}\right)\right\}
$$

Let

$$
F_{1}(p, \rho)=\frac{1}{768}\left\{7 p^{3}+8 p \rho\left(4-p^{2}\right)+32\left(4-p^{2}\right)+16 \rho^{2}(p-2)\left(4-p^{2}\right)\right\} .
$$

We assume that the upper bound occurs at the interior point of the rectangle $[0,2] \times[0,1]$. Differentiating (2.5) with respect to $\rho$, we get

$$
\frac{\partial F_{1}}{\partial \rho}=\frac{1}{768}\left\{8 p\left(4-p^{2}\right)+32 \rho(p-2)\left(4-p^{2}\right)\right\} .
$$

For $0<\rho<1$ and fixed $p \in(0,2)$, it can easily be seen that $\frac{\partial F_{1}}{\partial \rho}<0$. This shows that $F_{1}(p, \rho)$ is a decreasing function of $\rho$, which contradicts our assumption; therefore, $\max F_{1}(p, \rho)=$ $F_{1}(p, 0)=G_{1}(p)$. This implies that

$$
G_{1}^{\prime}(p)=\frac{1}{768}\left\{21 p^{2}-64 p\right\}
$$

and

$$
G_{1}^{\prime \prime}(p)=\frac{1}{768}\{42 p-64\}<0
$$

for $p=0$. Therefore $p=0$ is a point of maximum. Hence, we get the required result.

Lemma 2.6 If the function $f(z)=\sum_{n=1}^{\infty} a_{n} z^{n}$ belongs to the class $S L^{*}$, then

$$
\left|a_{2}\right| \leq 1 / 2, \quad\left|a_{3}\right| \leq 1 / 4, \quad\left|a_{4}\right| \leq 1 / 6, \quad\left|a_{5}\right| \leq 1 / 8 .
$$

These estimations are sharp. The first three bounds were obtained by Sokót [3] and the bound for $\left|a_{5}\right|$ can be obtained in a similar way.

Theorem 2.7 Let $f \in S L^{*}$ and of the form (1.1). Then

$$
\left|H_{3}(1)\right| \leq \frac{43}{576} .
$$


Proof Since

$$
H_{3}(1)=a_{3}\left(a_{2} a_{4}-a_{3}^{2}\right)-a_{4}\left(a_{4}-a_{2} a_{3}\right)+a_{5}\left(a_{1} a_{3}-a_{2}^{2}\right) .
$$

Now, using the triangle inequality, we obtain

$$
\left|H_{3}(1)\right| \leq\left|a_{3}\right|\left|a_{2} a_{4}-a_{3}^{2}\right|+\left|a_{4}\right|\left|a_{2} a_{3}-a_{4}\right|+\left|a_{5}\right|\left|a_{1} a_{3}-a_{2}^{2}\right| .
$$

Using the fact that $a_{1}=1$ with the results of Corollary 2.3, Theorem 2.4, Theorem 2.5 and Lemma 2.6, we obtain

$$
\left|H_{3}(1)\right| \leq \frac{43}{576}
$$

\section{Competing interests}

The authors declare that they have no competing interests.

\section{Authors' contributions}

MR and SNM jointly discussed and presented the ideas of this article. MR made the text file and all the communications regarding the manuscript. All authors read and approved the final manuscript.

\section{Author details}

${ }^{1}$ Department of Mathematics, G.C. University Faisalabad, Faisalabad, Pakistan. ${ }^{2}$ Department of Mathematics, COMSATS Institute of Information Technology, Defense Road Lahore, Pakistan.

\section{Acknowledgements}

The authors would like to thank the referees for helpful comments and suggestions which improved the presentation of the paper.

\section{Received: 15 February 2013 Accepted: 8 August 2013 Published: 28 August 2013}

\section{References}

1. Sokół, J, Stankiewicz, J: Radius of convexity of some subclasses of strongly starlike functions. Zesz. Nauk. Politech. Rzeszowskiej Mat. 19, 101-105 (1996)

2. Ali, RM, Cho, NE, Ravichandran, $\vee$, Kumar, SS: Differential subordination for functions associated with the lemniscate of Bernoulli. Taiwan. J. Math. 16(3), 1017-1026 (2012)

3. Sokół, J: Coefficient estimates in a class of strongly starlike functions. Kyungpook Math. J. 49(2), 349-353 (2009)

4. Noonan, JW, Thomas, DK: On the second Hankel determinant of areally mean $p$-valent functions. Trans. Am. Math. Soc. 223(2), 337-346 (1976)

5. Dienes, P: The Taylor Series. Dover, New York (1957)

6. Edrei, A: Sur les déterminants récurrents et les singularités d'une fonction donée por son développement de Taylor. Compos. Math. 7, 20-88 (1940)

7. Cantor, DG: Power series with integral coefficients. Bull. Am. Math. Soc. 69, 362-366 (1963)

8. Wilson, R: Determinantal criteria for meromorphic functions. Proc. Lond. Math. Soc. 4, 357-374 (1954)

9. Vein, R, Dale, P: Determinants and Their Applications in Mathematical Physics. Applied Mathematical Sciences, vol. 134. Springer, New York (1999)

10. Arif, M, Noor, Kl, Raza, M: Hankel determinant problem of a subclass of analytic functions. J. Inequal. Appl. (2012). doi:10.1186/1029-242X-2012-22

11. Arif, M, Noor, Kl, Raza, M, Haq, SW: Some properties of a generalized class of analytic functions related with Janowski functions. Abstr. Appl. Anal. 2012, Article ID 279843 (2012)

12. Bansal, D: Upper bound of second Hankel determinant for a new class of analytic functions. Appl. Math. Lett. 26(1), 103-107 (2013)

13. Janteng, A, Halim, SA, Darus, M: Coefficient inequality for a function whose derivative has a positive real part. J. Inequal. Pure Appl. Math. 7(2), Article 50 (2006)

14. Janteng, A, Halim, SA, Darus, M: Hankel determinant for starlike and convex functions. Int. J. Math. Anal. 1(13), 619-625 (2007)

15. Babalola, $\mathrm{KO}$ : On $\mathrm{H}_{3}$ (1) Hankel determinant for some classes of univalent functions. Inequal. Theory Appl. 6, 1-7 (2007)

16. Ma, WC, Minda, D: A unified treatment of some special classes of univalent functions. In: Li, Z, Ren, F, Yang, L, Zhang, S (eds.) Proceedings of the Conference on Complex Analysis (Tianjin, 1992), pp. 157-169. Int. Press, Cambridge (1994)

17. Grenander, U, Szegö, G: Toeplitz Forms and Their Applications. University of California Press, Berkeley (1958)

doi:10.1186/1029-242X-2013-412

Cite this article as: Raza and Malik: Upper bound of the third Hankel determinant for a class of analytic functions related with lemniscate of Bernoulli. Journal of Inequalities and Applications 2013 2013:412. 